\title{
Synthesis of Ordered Mesoporous Carbon Materials by Dry Etching
}

\author{
Winfried Nickel, ${ }^{[\mathrm{a}]}$ Martin Oschatz, ${ }^{[\mathrm{a}]}$ Soledad Rico-Francés, ${ }^{[\mathrm{b}]}$ Stefan Klosz, ${ }^{[\mathrm{c}]}$ Tim Biemelt, ${ }^{[\mathrm{a}]}$ Giovanni \\ Mondin, ${ }^{[\mathrm{a}]}$ Alexander Eychmüller, ${ }^{[\mathrm{c}]}$ Joaquín Silvestre-Albero, ${ }^{[\mathrm{b}]}$ and Stefan Kaskel ${ }^{[\mathrm{a}]_{*}}$
}

\begin{abstract}
A novel synthesis method for ordered mesoporous carbons is presented. Inverse replication of a silica template is achieved using the carbonization of sucrose within mesoporous KIT-6. Instead of liquid acid etching as in classical nanocasting, a novel dry chlorine etching is presented for template removal for the first time. The resulting ordered mesostructured carbon material outperforms carbons obtained by conventional hard templating with respect to high specific micro- and mesopore volumes (0.6 and $1.6 \mathrm{~cm}^{3} \mathrm{~g}^{-1}$, respectively) due to the presence of a hierarchical pore system. A high specific surface area of $1671 \mathrm{~m}^{2} \mathrm{~g}^{-1}$ is achieved, rendering this synthesis route as highly convenient method to produce ordered mesoporous carbons.
\end{abstract}

Porous carbon materials are crucial components in fields such as storage and filtration of gases ${ }^{[1]}$ or biomolecules, ${ }^{[2]}$ electrochemical energy storage ${ }^{[3]}$ water purification, ${ }^{[4]}$ and catalysis. ${ }^{[5]}$ Microporous carbons (pore width $<2 \mathrm{~nm}$ ) can be synthesized by different approaches. Materials obtained by the use of physical ${ }^{[6]}$ (carbon dioxide, steam) or chemical ${ }^{[7]}$ (zinc oxide, potassium hydroxide) activation techniques often lack well defined porosity, because the evolution of pores is hardly controllable. ${ }^{[8]}$ Zeolites can be used as template to achieve a defined and narrowly distributed porosity. ${ }^{[9]}$ A template-free approach is the treatment of silicon or transition metal carbides with hot chlorine gas to remove metal or semimetal atoms from the carbide matrix, resulting in microporous carbide-derived carbons with very narrow pore size distribution. ${ }^{[10]}$ However, several applications require hierarchical porosity, especially the simultaneous presence of larger pores to ensure rapid materials transport besides high specific surface area, i.e. hierarchical structures. ${ }^{[11]}$ Ryoo et al. pioneered the development of welldefined ordered mesoporous carbon (OMC) molecular sieves by nanocasting of the ordered mesoporous silica (OMS) MCM-48 (MCM: Mobil Composition of Matter) resulting in well-established OMCs designated as CMK-1 (CMK: Carbon Mesostructured by

[a] W. Nickel, M. Oschatz, G. Mondin, T. Biemelt, Prof. Dr. S. Kaskel Department of Inorganic Chemistry

TU Dresden

Bergstrasse 66, D-01069 Dresden (Germany)

Fax: (+)49 351 463-37287

E-mail: Stefan.Kaskel@chemie.tu-dresden.de

[b] S. Rico-Francés, Prof. Dr. J. Silvestre-Albero

Departamento de Química Inorgánica-Instituto Universitario de Materiales

Universidad de Alicante

Ap. 99, E-03080 Alicante (Spain)

[c] S. Klosz, Prof. Dr. A. Eychmüller

Department of Physical Chemistry

TU Dresden

Bergstrasse 66b, D-01069 Dresden (Germany)

Supporting information for this article is given via a link at the end of the document.
Kaist) ${ }^{[12]}$ Typically, a carbon precursor is infiltrated into the pore system of MCM-48, followed by carbonization and a separate template removal procedure using aqueous hydrofluoric acid or sodium hydroxide solutions followed by washing and drying (Scheme 1). This approach was further extended to other OMS materials with different geometries and pore sizes, such as SBA$15^{[13]}$ (SBA: Santa Barbara Amorphous) or KIT-6 ${ }^{[14]}$ (KIT: Korean Advanced Institute of Science and Technology), resulting in OMCs CMK- $3^{[15]}$ and CMK-8, ${ }^{[14]}$ respectively. Another procedure to obtain ordered mesoporous carbon materials by the use of soft templates such as block copolymers, acting as structure directing agent, was presented by Zhao et al. ${ }^{[16]}$ Despite the advantages of the soft templating approach, such as the avoidance of highly toxic hydrofluoric acid in the synthesis, this route is still complex. Besides the multi-step synthesis, these OMCs suffer from limited SSA usually below $1500 \mathrm{~m}^{2} \mathrm{~g}^{-1}$ for carbons obtained by nanocasting ${ }^{[15]}$ and $700 \mathrm{~m}^{2} \mathrm{~g}^{-1}$ for the soft template approach. ${ }^{[16]}$ Consequently, additional post-synthesis activation, ${ }^{[17]}$ wet-chemical etching ${ }^{[18]}$, or the carbide-derived carbon approach for micropore generation is applied to overcome these limitations. ${ }^{[19]}$

A new approach for oxide volatilization is dry etching by carbochlorination as recently demonstrated for the synthesis of carbons with disordered but uniform pore arrangement (equation 1): ${ }^{[20]}$

$$
\square \square \square 2+2 \square \square 2+2+\square \square \rightarrow \square \square \square \square 4+2 \square \square+\square \square
$$

As this reaction is the key step in the Kroll process for the production of titanium metal, ${ }^{[21]}$ the resulting carbon materials are denoted as Kroll-Carbons (KCs). KCs offer outstanding properties such as high specific surface areas close to $2000 \mathrm{~m}^{2} \mathrm{~g}^{-1}$ and mesopore volumes exceeding $3.0 \mathrm{~cm}^{3} \mathrm{~g}^{-1}$. The pore size is precisely controllable over a wide range from small mesopores to macropores by adjusting the particle size of $\mathrm{TiO}_{2} \cdot{ }^{[20]}$ The simultaneous presence of micropores in $\mathrm{KCs}$ is caused by the partial etching of carbon due to the evolution of CO. KCs show a high performance as cathode materials in lithium-sulfur batteries with very high active material loadings. So far, template removal using the reductive carbochlorination was successfully extended to alumina and silica as template. ${ }^{[22]}$ In spite of the fact that KCs obtained from fumed template particles offer uniform pore size, they are built by a void-windowtype mesopore system and might be less useful for applications in catalysis, where an ordered pore arrangement is largely required to achieve sufficient dispersion and advanced accessibility of catalytically active sites throughout the support. ${ }^{[23]}$

In the following, we describe for the first time the synthesis of Kroll-Carbons with an ordered arrangement of the mesopore system and narrow pore size distribution, as well as additional micropores, resulting in a hierarchical pore system. Ordered 
mesoporous Kroll-Carbons (OM-KCs, DUT-118) with cubic pore geometry are obtained by nanocasting of OMS with la3d symmetry (KIT-6, Supporting Information, Figure S1), followed by carbonization and subsequent template removal using the reductive carbochlorination reaction in chlorine atmosphere at $900{ }^{\circ} \mathrm{C}$ (Equation 2, Scheme 1).

$$
\square \square \square 2+2 \square \square 2+2+\square \square \rightarrow \square \square \square \square 4+2 \square \square+\square \square
$$

Compared to the carbon material (CMK-8) obtained by classical liquid phase template removal using highly toxic $\mathrm{HF}$ or $\mathrm{NaOH}$ solution, DUT-118 offer significantly improved textural parameters. In addition, the synthesis reported herein is a very straightforward route towards ordered mesoporous carbon materials as the number of synthesis steps can be reduced and the use of highly toxic hydrofluoric acid but also waste products are avoided. The reductive carbochlorination is a highly efficient process for template removal because it can be directly applied after carbonization and $\mathrm{SiCl}_{4}$ can be isolated as a highly pure and valuable byproduct.

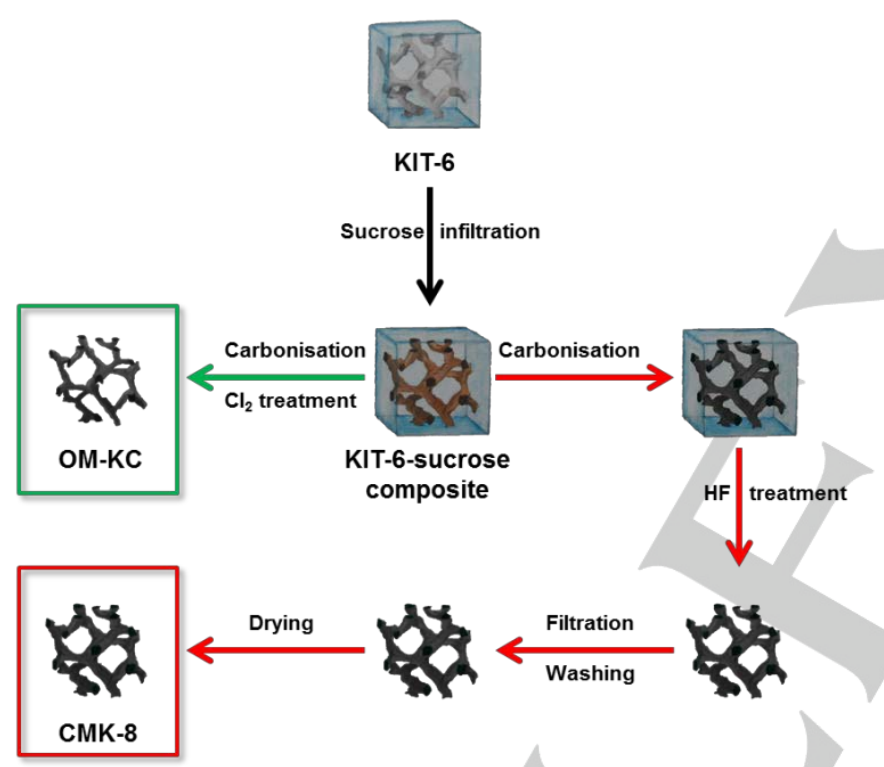

Scheme 1. Synthesis scheme of DUT-118 and CMK-8.

Electron dispersive X-ray spectroscopy (EDX) reveals quantitative removal of the silicon dioxide template even at moderate chlorination temperature applied $\left(900^{\circ} \mathrm{C}\right)$. The carbon content of DUT-118 is more than 99 atom\%, indicating the successful removal of the template due to the high reactivity of the silica/carbon nanocomposite, which is not expected for a physical or macroscale mixture of these compounds at comparable temperatures. ${ }^{[24]}$ Potential impurities such as silicon, oxygen or chlorine are below the detection limit. Further investigations using thermogravimetric measurements in air atmosphere (Supporting Information, Figure S2) show complete combustion of DUT-118 at temperatures above $600{ }^{\circ} \mathrm{C}$, proving the quantitative template removal by reductive carbochlorination and the absence of oxidic impurities.

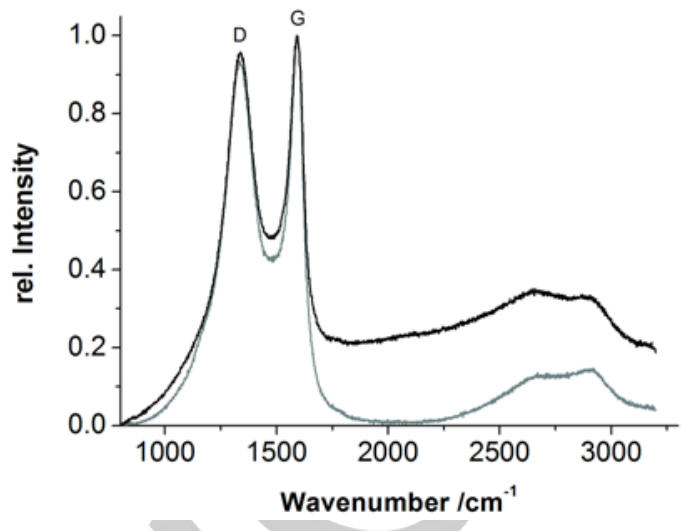

Figure 1. Raman spectra of DUT-118 (black) and CMK-8 (gray). Both display the D-band (at $\sim 1350 \mathrm{~cm}^{-1}$ ) and G-band $\left(\sim 1600 \mathrm{~cm}^{-1}\right)$, characteristic for amorphous carbons.

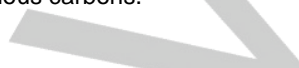

Despite the fact that DUT-118 is produced by a method that differs from conventional nanocasting approaches, the wall structure of DUT-118 and CMK-8 is comparable, as shown by Raman spectroscopy (Figure 1). Both spectra display a characteristic band at $\sim 1350 \mathrm{~cm}^{-1}$ (D-band), indicating a defect rich network structure as well as a low degree of graphitization due to the absence of pronounced peaks in the region from 2400 to $3000 \mathrm{~cm}^{-1}$, and a band at $\sim 1600 \mathrm{~cm}^{-1}$ (G-band). The intensity ratio of D to G-band of both DUT-118 and CMK-8 is 1.14, giving evidence that the microstructure of the carbon materials is only affected by the carbon source and synthesis temperature. It is noteworthy, that Raman spectra of Krollcarbons obtained from fumed silica as a template ${ }^{[22]}$ display similar shape but the intensity ratio from D- to G-band show higher values of 1.40 as well as lower full width at half maximum (FWHM) for the D1-band $\left(148 \mathrm{~cm}^{-1}\right.$ for DUT-118 and $121 \mathrm{~cm}^{-1}$ for $\mathrm{KC}$ derived from fumed silica), indicating a higher amount of amorphous $\mathrm{sp}^{2}$ carbon in DUT-118, possibly due to the confined space during synthesis within the pore system of KIT-6,

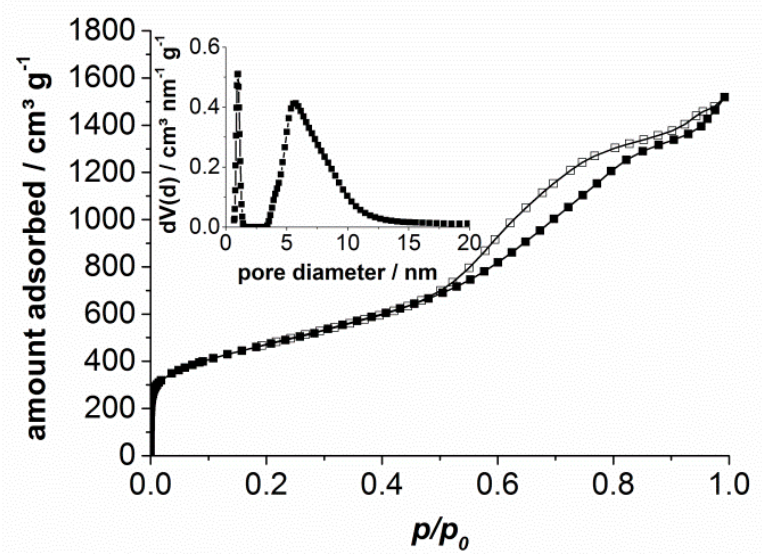

Figure 2. Nitrogen adsorption/desorption (filled symbols/empty symbols) isotherm (77 K) of DUT-118 and corresponding pore size distribution (inset). 
providing less room for the formation of a graphitic structure. ${ }^{[25]}$ Furthermore, FT-IR spectroscopy and TG-MS analysis were performed to investigate the presence of surface functional groups (Figures S3, S4). The IR spectra of DUT-118 and CMK-8 are comparable. Both samples show an intense band at around $3440 \mathrm{~cm}^{-1}$ that is characteristic for stretching vibrations of $-\mathrm{OH}$ groups that could arise from adsorbed water. The band at around $1630 \mathrm{~cm}-1$ is related to stretching vibrations of the $\mathrm{C}=\mathrm{O}$ group, giving evidence that oxygen-containing surface functional groups are present. This is further confirmed by TG-MS (Figure S4). Characteristic ions for water is detected at 75 and $300^{\circ} \mathrm{C}$, respectively. Evolution of $\mathrm{CO}_{2}$ is observed from $670^{\circ} \mathrm{C}$ up to $1000^{\circ} \mathrm{C}$ showing the presence of surface functional groups. Removal of the template during chlorine treatment leads to the simultaneous formation of micro- and mesopores. According to equation 1 , the formation of micropores is related to the evolution of CO resulting in an in-situ activation of DUT-118. The existence of micropores is observable in nitrogen physisorption measurement due to the high uptake at low relative pressures (Figure 2), resulting in a high specific BET surface area (SSA) of $1671 \mathrm{~m}^{2} \mathrm{~g}^{-1}$ and micropore volume of $0.60 \mathrm{~cm}^{3} \mathrm{~g}^{-1}$, calculated by the Dubinin-Radushkevich (DR) equation (Table 1). Although it is known that the DR approach overestimates the micropore volume if additional pore systems are present within the porous material ${ }^{[26]}$ it is clear that the micropore volume of DUT-118 is higher compared to CMK-8 (Table 1, Supporting Information, Figure S5). This is also observable at the semi-logarithmic plot of the isotherms (Supporting Information, Figure S6) where a higher nitrogen uptake for DUT-118 can be determined at low relative pressures related to micropore filling. QS-DFT pore size distribution (PSD) calculations using a kernel assuming combined slit- and cylindrical pores shows narrowly distributed micropores centered at $1 \mathrm{~nm}$ for both DUT-118 and CMK-8 (Figure 1 and Supporting Information, Figure S5, insets). While the formation of micropores within CMK- 8 is a random process originating from decomposition of the carbon precursor, the micropore system of DUT-118 is additionally related to the etching of carbon atoms out of the carbon structure during reaction, resulting in a larger volume of micropores. Additional experiments for the synthesis of DUT-118 were performed at a chlorination temperature of $1000^{\circ} \mathrm{C}$ (Figure S7). While the micropore volume is comparable for both materials $(0.60$ and $0.57 \mathrm{~cm}^{3} \mathrm{~g}^{-1}$, respectively), the total pore volume differs. DUT$118^{\circ} \mathrm{C}$ prepared at $1000^{\circ} \mathrm{C}$ has a lower total pore volume of 1.70 $\mathrm{cm}^{3} \mathrm{~g}^{-1}$ compared to DUT-118 prepared at $900^{\circ} \mathrm{C}$. Although higher reaction rates can be expected at higher temperature, higher shrinkage of the carbon framework during synthesis at $1000^{\circ} \mathrm{C}$ is likely to occur.

The unique micropore characteristics are further confirmed by $n$ nonane preadsorption studies. $n$-Nonane is known to selectively block the micropores within a porous material and allows to clearly distinguish them from mesopores as recently shown for other carbon materials with hierarchical structure. ${ }^{[27]}$ For both, DUT-118 and CMK-8, a lower amount of nitrogen can be adsorbed after $n$-nonane preadsorption. The shape of the isotherms is not affected by $n$-nonane loading, as can be seen by the hysteresis that occurs at the same relative pressure for the pristine samples and the samples loaded with hydrocarbon.
Table 1. Textural properties of DUT-118 and CMK-8.

\begin{tabular}{lllllll}
\hline Material & $\begin{array}{l}\mathrm{SSA}_{\mathrm{BET}}{ }^{[\mathrm{a}} \\
\left(\mathrm{m}^{2} \mathrm{~g}^{-1}\right)\end{array}$ & $\begin{array}{l}\mathrm{TPV}^{[\mathrm{b}]} \\
\left(\mathrm{cm}^{3} \mathrm{~g}^{-1}\right)\end{array}$ & $\begin{array}{l}\mathrm{MPV} \\
(\mathrm{DR})^{[\mathrm{c}]} \\
\left(\mathrm{cm}^{3} \mathrm{~g}^{-1}\right)\end{array}$ & $\begin{array}{l}\mathrm{MPV} \\
(\mathrm{DFT})^{[\mathrm{d}]} \\
\left(\mathrm{cm}^{3} \mathrm{~g}^{-1}\right)\end{array}$ & $\begin{array}{l}\mathrm{MPV} \\
\left(\mathrm{CO}_{2}\right)^{[\mathrm{e}]} \\
\left(\mathrm{cm}^{3} \mathrm{~g}^{-1}\right)\end{array}$ & $\begin{array}{l}\mathrm{MePV}^{[\mathrm{f}]} \\
\left(\mathrm{cm}^{3} \mathrm{~g}^{-1}\right)\end{array}$ \\
\hline $\begin{array}{l}\text { DUT- } \\
118\end{array}$ & 1671 & 2.20 & 0.60 & 0.21 & 0.16 & 1.60 \\
$\mathrm{CMK}^{\mathrm{C} 8}$ & 1308 & 1.20 & 0.47 & 0.20 & 0.14 & 0.73 \\
\hline
\end{tabular}

[a] SSA: Specific surface area; obtained using the BET equation in the relative pressure range $0.05-0.2 \mathrm{p} / \mathrm{p}_{0}[\mathrm{~b}] \mathrm{TPV}$ : Total pore volume, obtained at $\mathrm{p} / \mathrm{p}_{0}=0.95$ [c] MPV: Micropore volume (pore diameter $<2 \mathrm{~nm}$ ), obtained by Dubinin-Radushkevich calculations [d] Micropore Volume, obtained by QSDFT [e] Ultramicropore Volume (pore diameter $<0.7 \mathrm{~nm}$ ), obtained from $\mathrm{CO}_{2}$ adsorption isotherms using the Dubinin-Radushkevich equation [f] Mesopore Volume, obtained by subtraction of MPV (DR) from TPV

This indicates that micropores are selectively blocked and the mesopore system is not affected (Supporting Information, Figure S8). Both materials show a decrease in SSA of approximately $600 \mathrm{~m}^{2} \mathrm{~g}^{-1}$ (Supporting Information, Table S1). Further information of the ultramicropores present in the samples can be gained using $\mathrm{CO}_{2}$ adsorption at $0{ }^{\circ} \mathrm{C}$ up to ambient pressure (Supporting Information, Figure S9). Under these conditions, only narrow pores up to a diameter of $0.7 \mathrm{~nm}$ are filled. ${ }^{[28]}$ The higher ultramicropore volume of DUT-118 (Table 1) compared to CMK-8 gives further evidence that the microporosity of DUT-118 is more pronounced.

The distinct hysteresis loop in the nitrogen physisorption isotherm of DUT-118 at relative pressures ranging from 0.5 to 0.8 is attributed to the formation of mesopores during the reductive carbochlorination. Pore widening occurs due to the consumption of the carbon, resulting in larger mesopores compared to CMK-8, ranging from $\sim 4$ to $\sim 13 \mathrm{~nm}$ with a maximum centered at $5.7 \mathrm{~nm}$. The good agreement between the experimental data and QS-DFT kernel (Supporting Information, Figure S10) shows that this method calculates a reliable PSD. Furthermore it is observable that PSDs obtained by QS-DFT calculations using adsorption and desorption branches of the isotherm are comparable, indicating that desorption in the mesopore system takes places under equilibrium conditions (Supporting Information, Figure S11). Cavitation or pore blocking phenomena can be ruled out indicating the unhindered accessibility of the mesopore system of DUT-118 for the probe molecules.

The total pore volume of DUT-118 (obtained from nitrogen physisorption isotherms at $\mathrm{p} / \mathrm{p}_{0}=0.95$ ) is $2.2 \mathrm{~cm}^{3} \mathrm{~g}^{-1}$ and thus nearly twice as high as that of CMK-8 $\left(1.2 \mathrm{~cm}^{3} \mathrm{~g}^{-1}\right)$. Due to the reductive carbochlorination mechanism applied for template removal in the synthesis of DUT-118, approximately $50 \%$ of the carbon mass is consumed during the reaction, which is mostly responsible for the higher pore volume. Further evidence can be gained by $n$-nonane preadsorption as well. DUT-118 still offers a higher specific surface area as well as specific pore volume compared to CMK-8 after $n$-nonane loading due to the higher amount of mesopores present in DUT-118 (Supporting Information, Table S1).

Despite the pore widening due to consumption of the template during reaction, the resulting DUT-118 shows a long range order as evidenced by low angle $\mathrm{X}$-ray diffraction (XRD) 
and transmission electron microscopy (TEM). Low angle XRD pattern (Supporting Information, Figure S12) of DUT-118 exhibits a peak at 20 1.01, indicating a mesoscopic ordering. The low angle XRD pattern of CMK-8 shows a peak at $2 \theta \sim 1.04$. The unit cell size of DUT-118 and CMK-8 can be estimated to $21.4 \mathrm{~nm}$ and $20.8 \mathrm{~nm}$, respectively. Both values are comparable, indicating that the mesostructure of DUT-118 is only slightly affected during synthesis. TEM images show a: periodic arrangement of DUT-118 over a long range, proving its ordered structure (Figure 3). It is noted, that if a template with different geometry (e.g. SBA-15 with hexagonally ordered cylindrical mesopores interconnected by randomly distributed micropores) is used instead of the KIT- 6 with three-dimensional gyroidal pore structure, the resulting carbon material exhibits high SSA but no long range order (Figure S13, S14).
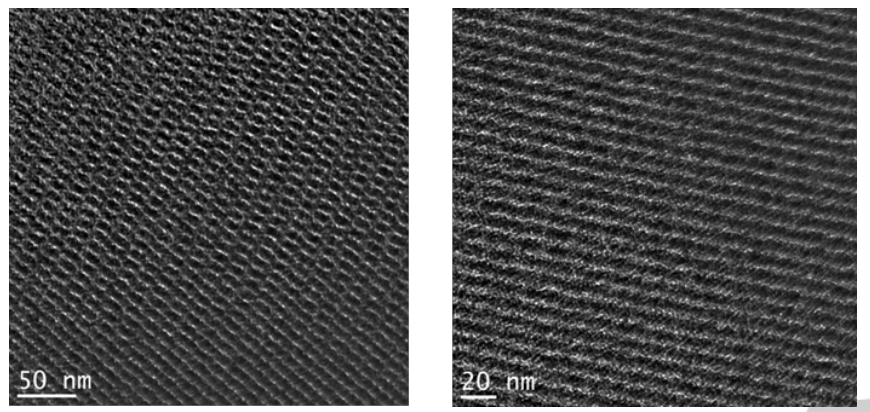

Figure 3. TEM images of DUT-118

In summary, we have presented for the first time the synthesis of ordered mesoporous Kroll-Carbons as a new class of highly porous carbons with hierarchical pore structure. This synthetic route is highly versatile for the production of ordered mesoporous carbon materials with well-defined mesoporosity and long range order. Despite the fact that toxic chlorine gas is used for this synthesis, the reductive carbochlorination is a highly efficient process because it can be directly applied after carbonization and $\mathrm{SiCl}_{4}$ can be recovered as useful byproduct. Moreover, it is an established large scale industrial process in the titanium industry with high space time yield that does not require time- and volume-intensive wet chemical processing. DUT-118 has high SSA of $1671 \mathrm{~m}^{2} \mathrm{~g}^{-1}$ as well as high micro- and mesopore volumes of 0.6 and $1.6 \mathrm{~cm}^{3} \mathrm{~g}^{-1}$, respectively, significantly exceeding ordered mesoporous carbon materials obtained by classical nanocasting approaches based on wet chemical template removal. This process needs less reaction steps and leads to the simultaneous evolution of micro- and mesopores, i.e. the formation of a hierarchical pore system that is not present in classical mesoporous materials. This new class of hierarchical mesoporous carbons will be valuable for applications such as catalysis or electrochemical energy storage.

\section{Acknowledgements}

Financial support from the Deutsche Forschungsgemeinschaft is gratefully acknowledged. We thank F. Drache for drawing the 3D graphic (Scheme 1).

Keywords: carbon $\cdot$ mesoporous materials $\bullet$ hierarchical pore system $\bullet$ adsorption $\bullet$ nanoporous carbon

[1] a) M. Oschatz, L. Borchardt, M. Thommes, K. A. Cychosz, I. Senkovska, N. Klein, R. Frind, M. Leistner, V. Presser, Y. Gogotsi, S. Kaskel, Angew. Chem. 2012, 124, 7695-7698; Angew. Chem. Int. Ed. 2012, 51, 7577-7580; b) A. Silvestre-Albero, S. RicoFrancés, F. Rodríguez-Reinoso, A. M. Kern, M. Klumpp, B. J. M. Etzold, J. Silvestre-Albero, Carbon 2013, 59, 221-228.

[2] a) S. Yachamaneni, G. Yushin, S.-H. Yeon, Y. Gogotsi, C. Howell, S. Sandeman, G. Phillips, S. Mikhalovsky, Biomaterials 2010, 31, 4789-4794; b) F. Hippauf, D. Lunow, L. Borchardt, T. Henle, S Kaskel, Carbon 2014, 77, 191-198.

[3] a) L. Borchardt, M. Oschatz, S. Kaskel, Mater. Horiz. 2014, 1, 157 168; b) P. G. Bruce, S. A. Freunberger, L. J. Hardwick, J.-M. Tarascon, Nat. Mater: 2012, 11, 19-29; c) P. Simon, Y. Gogotsi, Nat. Mater. 2008, 7, 845-854.

[4] S. Porada, L. Borchardt, M. Oschatz, M. Bryjak, J. S. Atchison, K. J. Keesman, S. Kaskel, P. M. Biesheuvel, V. Presser, Energy Environ. Sci. 2013, 6, 3700-3712.

[5] a) L. Borchardt, F. Hasché, M. R. Lohe, M. Oschatz, F. Schmidt, E. Kockrick, C. Ziegler, T. Lescouet, A. Bachmatiuk, B. Büchner, D. Farrusseng, P. Strasser, S. Kaskel, Carbon 2012, 50, 1861-1870; b) Y. Zhao, L. Yang, S. Chen, X. Wang, Y. Ma, Q. Wu, Y. Jiang, W. Qian, Z. Hu, J. Am. Chem. Soc. 2013, 135, 1201-1204.

[6] H. Marsh, F. Rodríguez-Reinoso in Activated Carbon, Elsevier Science Ltd, Oxford, 2006, pp. 243-321.

[7] H. Marsh, F. Rodríguez-Reinoso in Activated Carbon, Elsevier Science Ltd, Oxford, 2006, pp. 322-365.

K. P. Gadkaree, M. Jaroniec, Carbon 2000, 38, 983-993.

[9] T. Kyotani, T. Nagai, S. Inoue, A. Tomita, Chem. Mater. 1997, 9 , 609-615.

[10] a) Y. Gogotsi, A. Nikitin, H. Ye, W. Zhou, J. E. Fischer, B. Yi, H. C. Foley, M. W. Barsoum, Nat. Mater. 2003, 2, 591-594; b) V. Presser, M. Heon, Y. Gogotsi, Adv. Funct. Mater. 2011, 21, 810-833.

[11] J. Lee, J. Kim, T. Hyeon, Adv. Mater. 2006, 18, 2073-2094.

[12] a) R. Ryoo, S. H. Joo, M. Kruk, M. Jaroniec, Adv. Mater. 2001, 13 677-681; b) R. Ryoo, S. H. Joo, S. Jun, J. Phys. Chem. B 1999, 103, 7743-7746.

[13] D. Zhao, J. Feng, Q. Huo, N. Melosh, G. H. Fredrickson, B. F Chmelka, G. D. Stucky, Science 1998, 279, 548-552.

[14] T.-W. Kim, F. Kleitz, B. Paul, R. Ryoo, J. Am. Chem. Soc. 2005, 127, 7601-7610.

[15] S. Jun, S. H. Joo, R. Ryoo, M. Kruk, M. Jaroniec, Z. Liu, T. Ohsuna O. Terasaki, J. Am. Chem. Soc. 2000, 122, 10712-10713.

Y. Meng, D. Gu, F. Zhang, Y. Shi, H. Yang, Z. Li, C. Yu, B. Tu, D. Zhao, Angew. Chem. 2005, 117, 7215-7221; Angew. Chem. Int. Ed. 2005, 44, 7053-7059.

[17] a) D. Wu, Y. Liang, X. Yang, C. Zou, Z. Li, G. Lv, X. Zeng, R. Fu Langmuir 2008, 24, 2967-2969; b)K. Xia, Q. Gao, C. Wu, S. Song M. Ruan, Carbon 2007, 45, 1989-1996.

[18] R. Liu, Y. Shi, Y. Wan, Y. Meng, F. Zhang, D. Gu, Z. Chen, B. Tu, D. Zhao, J. Am. Chem. Soc. 2006, 128, 11652-11662.

P. Krawiec, E. Kockrick, L. Borchardt, D. Geiger, A. Corma, S. Kaskel, J. Phys. Chem. C 2009, 113, 7755-7761.

[20] M. Oschatz, S. Thieme, L. Borchardt, M. R. Lohe, T. Biemelt, J. Bruckner, H. Althues, S. Kaskel, Chem. Commun. 2013, 49, 5832 5834.

[21] W. Kroll, US 2205854 A 1940

[22] M. Oschatz, S. Boukhalfa, W. Nickel, J. T. Lee, S. Klosz, L. Borchardt, A. Eychmuller, G. Yushin, S. Kaskel, J. Mater. Chem. A 2014, 2, 5131-5139.

[23] a) M. Wang, X. Wang, Q. Yue, Y. Zhang, C. Wang, J. Chen, H. Cai, H. Lu, A. A. Elzatahry, D. Zhao, Y. Deng, Chem. Mater. 2014, 26, 3316-3321; b) K.-S. Ha, G. Kwak, K.-W. Jun, J. Hwang, J. Lee, Chem. Commun. 2013, 49, 5141-5143.

E. S. M. Seo, M. Andreoli, R. Chiba, J. Mater. Process. Technol. 2003, 141, 351-356.

K. Faber, F. Badaczewski, M. Oschatz, G. Mondin, W. Nickel, S. Kaskel, B. M. Smarsly, J. Phys. Chem. C 2014, 118, 15705-15715. P. J. M. Carrott, F. L. Conceição, M. M. L. R. Carrott, Carbon 2007 $45,1310-1313$

a) A. Silvestre-Albero, M. Gonçalves, T. Itoh, K. Kaneko, M. Endo, M. Thommes, F. Rodríguez-Reinoso, J. Silvestre-Albero, Carbon 
2012, 50, 66-72; b) M. Oschatz, L. Borchardt, S. Rico-Francés, F.

Rodríguez-Reinoso, S. Kaskel, J. Silvestre-Albero, Langmuir 2013

29, 8133-8139.
[28]

J. Silvestre-Albero, A. Silvestre-Albero, F. Rodríguez-Reinoso, M. Thommes, Carbon 2012, 50, 3128-3133. 


\section{COMMUNICATION}
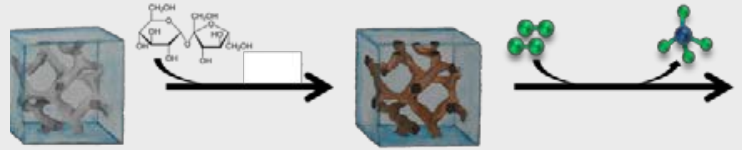

KIT-6-sucrose composite

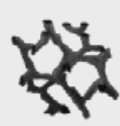

OM-KC

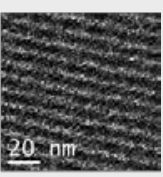

A solvent free template removal method for the synthesis of ordered mesoporous carbons is discovered, using a carbochlorination reaction. This process needs less reaction steps and introduces simultaneously micro- and mesopores, resulting in a hierarchical pore system outperforming established mesoporous carbon materials.
Winfried Nickel, ${ }^{[a]}$ Martin Oschatz, ${ }^{[a]}$

Soledad Rico-Francés, ${ }^{[b]}$ Stefan

Klosz, ${ }^{[c]}$ Tim Biemelt, ${ }^{[a]}$ Giovanni Mondin, ${ }^{[a]}$ Alexander Eychmüller, ${ }^{[c]}$

Joaquín Silvestre-Albero, ${ }^{[b]}$ and Stefan

Kaskel $^{[a] *}$

Page No. - Page No.

Synthesis of Ordered Mesoporous Carbon Materials by Dry Etching 